\title{
Cost-Effectiveness Analysis of Diagnostic Tests for Para-Aortic Lymph Node Detection in Locally Advanced Cervical Cancer
}

\author{
Kanyarat Katanyoo iD 1,2 \\ Usa Chaikledkaew ${ }^{3,4}$ \\ Montarat Thavorncharoensap ${ }^{3,4}$ \\ Arthorn Riewpaiboon (iD) ${ }^{3}$ \\ 'Social, Economic and Administrative \\ Pharmacy (SEAP) Graduate Program, \\ Department of Pharmacy, Faculty of \\ Pharmacy, Mahidol University, Bangkok, \\ Thailand; ' 2 Department of Radiology, \\ Faculty of Medicine Vajira Hospital, \\ Navamindradhiraj University, Bangkok, \\ Thailand; ${ }^{3}$ Social and Administrative \\ Pharmacy Division, Department of \\ Pharmacy, Faculty of Pharmacy, Mahido \\ University, Bangkok, Thailand; ${ }^{4}$ Mahidol \\ University Health Technology \\ Assessment (MUHTA) Graduate \\ Program, Mahidol University, Bangkok, \\ Thailand
}

\begin{abstract}
Purpose: We aimed to determine the cost-effectiveness of diagnostic tests, ie, computed tomography (CT), magnetic resonance imaging (MRI), and fluorine-18-fluorodeoxyglucose positron emission tomography/computed tomography (PET/CT) for para-aortic lymph node detection (PALND), in locally advanced cervical cancer (LACC) patients (stages IB3-IVA) with or without laparoscopic lymphadenectomy (LL) compared with no investigation (NoIx) based on provider and societal perspectives during 5 years.
\end{abstract}

Patients and Methods: Hybrid decision tree and Markov models were conducted to compare the cost and utility of six interventions including: 1) CT without LL, 2) CT with LL, 3) MRI without LL, 4) MRI with LL, 5) PET/CT without LL, and 6) PET/CT with LL compared with NoIx. All clinical parameters were obtained from published studies. Costs were presented in year 2019 values. Direct medical costs were retrieved from hospital database, while direct non-medical costs and utility were collected from interviewing 194 LACC patients during June to December 2019. One-way and probabilistic sensitivity analysis were used to investigate parameter uncertainties.

Results: Total costs of NoIx were $\$ 8026$ and $\$ 11,444$ from provider and societal perspectives, respectively, and quality-adjusted life year (QALY) was 3.70. NoIx was more effective and less costly. When six strategies were compared with NoIx, more additional costs were shown with \$1835, \$1735, \$2022, \$1987, \$4002, and \$4176 for CT without LL, CT with LL, MRI without LL, MRI with LL, PET/CT without LL, and PET/CT with LL, whereas QALYs were decreased with $0.07,0.08,0.07,0.08,0.05$, and 0.07 , respectively. Sensitivity analyses strengthened the benefit of NoIx. The most significant parameter was treatment outcomes of patients with PALN metastasis.

Conclusion: NoIx or receiving basic clinical staging was a dominant option when compared with CT, MRI, and PET/CT for PALND before providing the treatment for LACC patients Keywords: cost-utility analysis, stage IIIC2 cervical cancer, computed tomography, magnetic resonance imaging, PET/CT

\section{Introduction}

In developing countries such as Thailand, cervical cancer is still being threatened cancer for women, followed by breast cancer. In 2018, the estimated number of new cervical cancer patients and deaths was 8622 and 5015, respectively. It means that approximately one Thai woman dies every two hours from cervical cancer. ${ }^{1}$ The incidence of this cancer is varied across countries depending on the level of economic performance. ${ }^{2}$ The most important prognostic factor for cervical cancer is stage of disease. In 2018, the International Federation of Gynecology and
Correspondence: Usa Chaikledkaew Social and Administrative Pharmacy Division, Department of Pharmacy, Faculty of Pharmacy, Mahidol University, 447 Sri-Ayudhaya Road, Bangkok, Thailand

Tel +662-644-8678 ext 5317

Fax +662-644-8694

Email usa.chi@mahidol.ac.th 
Obstetrics (FIGO) staging system added the new stages, ie, stages IIIC1 and IIIC2 for disease at pelvic lymph node (PLN) and para-aortic lymph node (PALN), respectively. ${ }^{3}$ The incidence of metastatic disease to PALN is dependent on clinical staging at the first diagnosis. For locally advanced cervical cancer (LACC) patients or stages IB3IVA, the overall incidence of disease at PALN was about $16 \%{ }^{4}$

The gold standard for PALN detection (PALND) is histopathology by surgical operation. ${ }^{4,5}$ However, special imaging, ie, computed tomography (CT), magnetic resonance imaging (MRI), and fluorine-18-fluorodeoxyglucose positron emission tomography/computed tomography (PET/CT), is easier to perform than surgical staging and preferred by physicians for PALND. Currently, according to the FIGO 2018, the ideal staging is special imaging, ie, CT, MRI, PET/CT, or pathological approach at enlarged pelvic lymph nodes and para-aortic lymph nodes, which has been applied in developed countries which have unconstrained resources and can adopt new staging systems. ${ }^{3}$ These images and pathological approach were also used to establish stages IIIC1r (imaging), IIIC1p (pathology), IIIC2r, and IIIC2p according to the FIGO 2018 staging system. ${ }^{3}$ However, these special investigations are not mandatory, if there are limitations to their accessibility, ie, no equipment such as MRI and PET/CT in hospitals, long waiting time for investigations, and lack of skills for laparoscopic lymphadenectomy (LL). Therefore, based on the FIGO staging 2018, basic clinical staging, ie, physical examination, pelvic examination, and biopsy of cervical tumor, has also been accepted to be used as the standard of care in lowresource conditions. ${ }^{3}$

The limitation of each image is their accuracy including sensitivity (true positive rate; TP rate) and specificity (true negative rate; $\mathrm{TN}$ rate), thus some errors of these assessments, ie, understaging (false negative; FN) or overstaging (false positive; FP), cannot be avoided. Moreover, previous studies revealed more survival benefit from pathological diagnosis than radiological diagnosis. ${ }^{6-8}$ For laparoscopic para-aortic lymphadenectomy, two techniques have been described, ie, transperitoneal LL and extraperitoneal LL, and there was no difference between these two techniques regarding complications after surgery. ${ }^{9}$

The importance of PALN metastasis detection is to increase the curative chance of treatment for stage IIIC2 cervical cancer patients. Nowadays, there is no standard treatment for patients who had suspected disease at PALN by diagnostic tests. Extended-field radiation therapy (EFRT) is recommended for patients who have evidence of disease at
PALN. ${ }^{10,11}$ A range of treatment outcomes of 5-year diseasefree survival (DFS) and 5-year overall survival (OS) of 18\% to $45 \%$ and $30 \%$ to $70 \%$, respectively, has been reported, ${ }^{12-19}$ and increase of some late side effects (SEs) was unavoidable. $^{20,21}$ Therefore, the benefit of screening disease at PALN was still questionable. Apart from OS, another treatment outcome that is important for patients is quality of life from the consequence of treatment. Moreover, cost of investigations, treatments, and complications due to the treatment should be of concern. Therefore, it is important to investigate the cost and outcomes of all diagnostic tests for PALND, as physicians usually order these investigations as a routine practice without concerns about these aspects.

Until recently, there has been no previous economic evaluation evidence regarding the value for money of such special diagnostic tests in developing countries such as Thailand, yet. Therefore, the purpose of this study was to determine the costeffectiveness of diagnostic tests, ie, CT, MRI, and PET/CT for PALND in LACC patients (stages IB3-IVA) with or without LL compared with NoIx based on provider and societal perspectives during 5 years at the national level.

\section{Materials and Methods Economic Evaluation}

Cost-utility analysis was applied to compare the costs and outcomes of diagnostic tests for PALND before providing definite treatment. Hybrid decision tree and Markov models were conducted based on healthcare provider and societal perspectives. The time horizon of this study was 5 years which assumed as cancer-free absolutely with a cycle length of 6 months. Target populations included LACC patients with stages IB3-IVA. Three diagnostic tests, ie, CT, MRI, or PET/CT, were compared with NoIx. If PALN was enlarged from diagnostic tests, laparoscopic lymphadenectomy (LL) was the component of comprehensive investigation for some physicians to confirm a positive finding. In order to include more variation of practice, LL was the option in this study in case imaging was positive. The compared interventions were as follows: (1) CT without LL, (2) CT with LL if image was positive, (3) MRI without LL, (4) MRI with LL if image was positive, (5) PET/CT without LL, (6) PET/CT with LL if image was positive, and (7) NoIx. After patients had received clinical staging by pelvic examination and were classified as stages IB3-IVA, concurrent chemoradiation therapy (CCRT) was provided. Standard CCRT was composed of external beam radiation therapy at the whole pelvis by two- 
dimensional technique at 56 Gy (28 fractions) with weekly cisplatin $40 \mathrm{mg} / \mathrm{m}^{2}$ for six cycles and high dose-rate brachytherapy four times. The results were presented as the incremental cost-effectiveness ratio (ICER) calculated by a difference in costs divided by a difference in qualityadjusted life years (QALYs) or number of cases detected between each diagnostic test and NoIx.

\section{Economic Evaluation Model}

Decision tree and Markov models were developed using Microsoft Office Excel 2010 (Microsoft Corp., Redmond, WA, USA). Figure 1 illustrates a decision tree model for PALND without LL. After LACC patients received each diagnostic test, they might have either PALN enlargement $(>10 \mathrm{~mm}$ in short axis measurement) or suspected metastatic disease $^{22}$ or no PALN enlargement $(\leq 10 \mathrm{~mm}$ in short axis measurement). For those without PALN enlargement having TN or FN test results, they would receive CCRT. On the other hand, for those with PALN enlargement having TP or FP test results, they would receive EFRT by three-dimensional conformal radiation therapy (3D-CRT) with the total dose of 60 Gy in 30 fractions after CCRT at the whole pelvis.

Figure 2 shows a decision tree model for PALND with LL. If either a FP or TP test result was found, LL would be performed. If the TP test result confirmed that there were cancer cells at this node, patients had to undergo EFRT

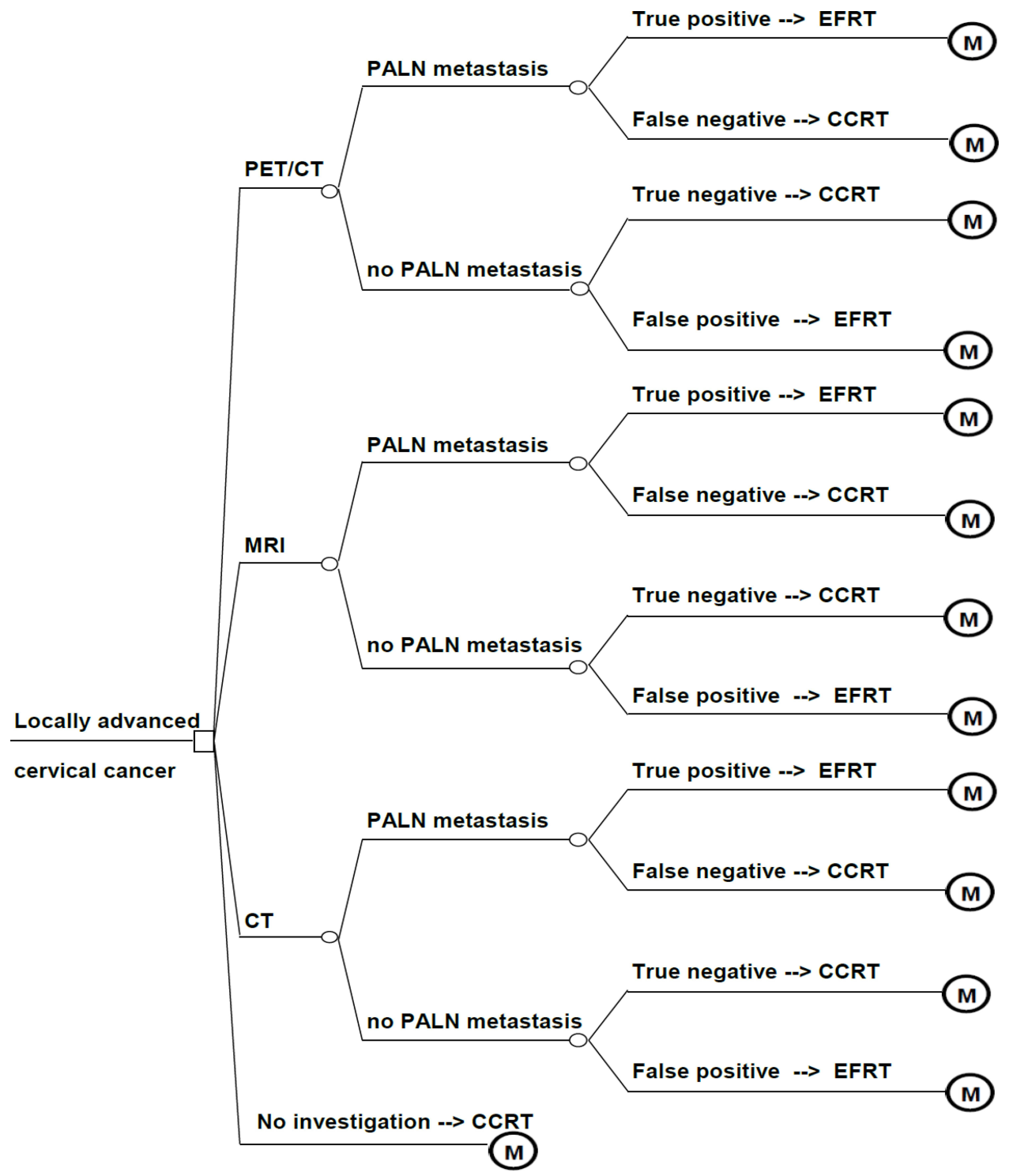

Figure I Decision tree of para-aortic lymph node detection with the first approach: without laparoscopic lymphadenectomy.

Abbreviations: CT, computed tomography; MRI, magnetic resonance imaging; PET/CT, positron emission tomography/computed tomography; PALN, para-aortic lymph node; CCRT, concurrent chemoradiation; EFRT, extended-field radiation therapy; M, Markov model. 


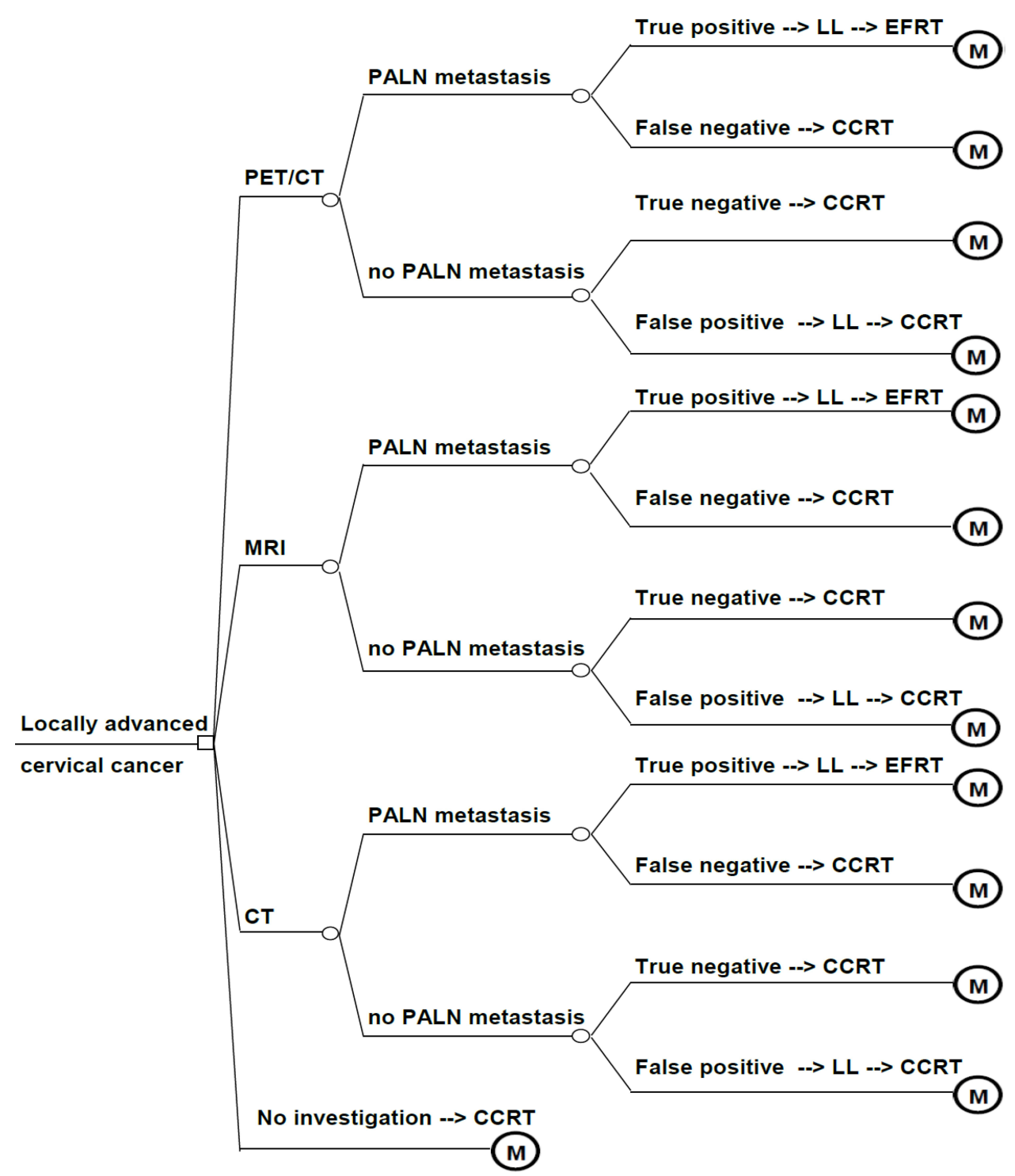

Figure 2 Decision tree of para-aortic lymph node detection with the second approach: laparoscopic lymphadenectomy.

Abbreviations: CT, computed tomography; MRI, magnetic resonance imaging; PET/CT, positron emission tomography/computed tomography; PALN, para-aortic lymph node; CCRT, concurrent chemoradiation; EFRT, extended-field radiation therapy; LL, laparoscopic lymphadenectomy; M, Markov model.

with the total dose of $50.4 \mathrm{~Gy}$ in 28 fractions. If either TN or FN was found, CCRT would be given. We assumed that the patients finishing the treatment would be followed up every 3 months for the first two years and then every 6 months for the third to fifth years.

After LACC patients completed treatment with CCRT or CCRT plus EFRT from decision trees, the patients' costs and outcomes were estimated using a Markov model consisting of four health states including no disease or disease-free, disease progression (local recurrence or distant metastasis), severe late SEs in terms of gastrointestinal (GI) and genitourinary (GU) systems, and death states (Figure 3). All patients would start from no disease state, and could stay at the same health state or move to progression, late SEs, or death. Our model assumptions were as follows: 1) all patients had no metastatic disease to other organs except PALN at the time of diagnosis; 2) no delayed treatment such as CCRT or EFRT due to complications of LL; 3) two-dimensional radiation therapy at whole pelvis was applied; 4) when disease was progressive, recurrence, or distant metastasis, these patients had median survival time about 18 months by palliative carboplatin plus paclitaxel for 6 cycles and supportive care. ${ }^{23}$ This study did not include bevacizumab for systemic treatment, because this targeted therapy cannot be reimbursed for treatment in stage IVB or metastatic or recurrent cervical cancer patients in Thailand. 


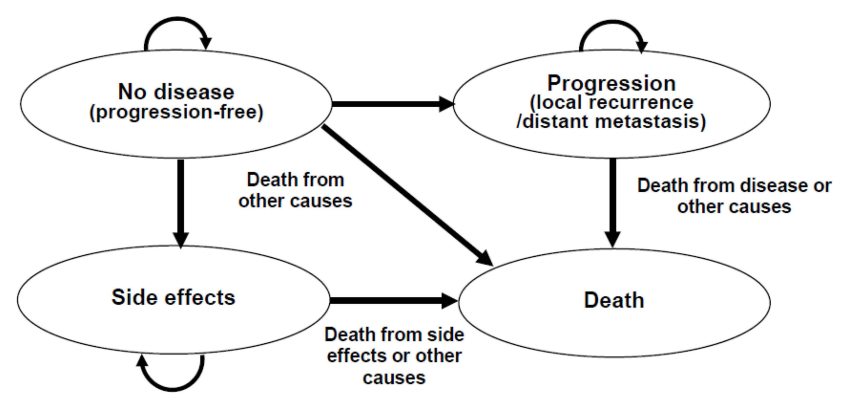

Figure 3 The schematic diagram of the Markov model.

\section{Model Parameters}

Clinical Parameters

Table 1 presents all parameters used in this study. All clinical parameters in the decision tree model, ie, incidence of PALN metastasis, ${ }^{4}$ diagnostic accuracy for PALND from CT, MRI, and PET/CT, ${ }^{24}$ were obtained from landmark clinical studies. The probabilities of clinical outcomes for LACC patients with NoIx were obtained from a Thai study. ${ }^{25}$ The probabilities of 5 -year DFS, OS for patients with disease progression after completing treatment, and severe late SEs were extracted from international published studies. ${ }^{17,19,23,26}$ From the study of Lai et al, ${ }^{12}$ it was assumed that late GI and GU SEs for NoIx, EFRT without LL, and CCRT after LL were identical. Age-specific population mortality rate was retrieved from the World Health Organization (WHO) ${ }^{27}$

\section{Cost Parameters}

According to a societal perspective, direct medical costs (DMCs) and direct non-medical costs (DnMCs) were included, ${ }^{28}$ whereas only DMC was considered based on healthcare provider's perspective. DMC included the costs of all laboratory tests, diagnostic tests, treatment modalities, cost of management for severe late SEs during 5 years, cost of palliative chemotherapy (carboplatin plus paclitaxel for 6

Table I Clinical Parameters

\begin{tabular}{|c|c|c|c|c|}
\hline Parameters & \multicolumn{3}{|c|}{ Mean (95\% Confidence Interval) } & Reference \\
\hline Incidence of PALN (stage IB-IVA) & \multicolumn{3}{|c|}{$0.16(0.13-0.18)$} & [4] \\
\hline Diagnostic test accuracy & CT & MRI & PET/CT & \\
\hline True negative (TN) & $0.91(0.89-0.93)$ & $0.93(0.91-0.95)$ & $0.97(0.98-0.96)$ & [24] \\
\hline True positive (TP) & $0.57(0.47-0.67)$ & $0.54(0.44-0.64)$ & $0.66(0.57-0.75)$ & [24] \\
\hline False negative (FN) & $0.43(0.33-0.53)$ & $0.46(0.36-0.56)$ & $0.34(0.25-0.43)$ & [24] \\
\hline False positive (FP) & $0.09(0.07-0.11)$ & $0.07(0.05-0.09)$ & $0.03(0.02-0.04)$ & [24] \\
\hline \multicolumn{4}{|c|}{ Survival data at 5 years (disease-free survival) } & \\
\hline No investigation $\rightarrow$ CCRT & & \multicolumn{2}{|c|}{$0.63(0.55-0.7 I)$} & {$[25]$} \\
\hline \multicolumn{2}{|c|}{ Diagnostic test with no LL when suspected disease at PALN } & & \\
\hline - no suspected disease at PALN (TN) & CCRT & \multicolumn{2}{|c|}{$0.65(0.57-0.73)$} & [26] \\
\hline - no suspected disease at PALN (FN) & CCRT & \multicolumn{2}{|c|}{$0.06(0.02-0.10)$} & [23] \\
\hline - suspected disease at PALN (TP and & $\rightarrow$ EFRT & \multicolumn{2}{|c|}{$0.46(0.32-0.60)$} & [17] \\
\hline \multicolumn{5}{|c|}{ Diagnostic test with LL when suspected disease at PALN } \\
\hline - disease (TP) $\rightarrow$ EFRT & & \multicolumn{2}{|c|}{$0.29(0.16-0.42)$} & [19] \\
\hline - no disease (FP) $\rightarrow$ CCRT & & \multicolumn{2}{|c|}{$0.65(0.57-0.73)$} & [26] \\
\hline \multicolumn{2}{|c|}{ Overall survival rate after when disease progression } & \multicolumn{2}{|c|}{$0.10(0.05-0.15)$} & [23] \\
\hline \multicolumn{5}{|c|}{ Severe late side effect rates (grades 3-5) } \\
\hline \multicolumn{2}{|c|}{ - gastrointestinal system from CCRT $\pm \mathrm{LL}$} & \multicolumn{2}{|c|}{$0.06(0.02-0.10)$} & [26] \\
\hline \multicolumn{2}{|c|}{ - genitourinary system from CCRT \pm LL } & \multicolumn{2}{|c|}{$0.17(0.11-0.23)$} & [26] \\
\hline \multicolumn{2}{|l|}{ - gastrointestinal system from EFRT } & \multicolumn{2}{|c|}{$0.06(0.02-0.10)$} & [26] \\
\hline \multicolumn{2}{|l|}{ - genitourinary system from EFRT } & \multicolumn{2}{|c|}{$0.17(0.11-0.23)$} & [26] \\
\hline \multicolumn{2}{|l|}{ - gastrointestinal system from LL + EFRT } & \multicolumn{2}{|c|}{$0.11(0.02-0.20)$} & [19] \\
\hline \multicolumn{2}{|l|}{ - genitourinary system from LL + EFRT } & \multicolumn{2}{|c|}{$0.19(0.08-0.30)$} & [19] \\
\hline \multicolumn{2}{|l|}{ - death from CCRT $\pm \mathrm{LL}$} & \multicolumn{2}{|c|}{$0.01(0-0.03)$} & [26] \\
\hline \multicolumn{2}{|l|}{ - death from CCRT + EFRT } & \multicolumn{2}{|c|}{$0.01(0-0.02)$} & [17] \\
\hline \multicolumn{2}{|l|}{ - death from LL + EFRT } & \multicolumn{2}{|c|}{$0.02(0-0.06)$} & [19] \\
\hline
\end{tabular}

Abbreviations: PALN, para-aortic lymph node; FN, false negative; FP, false positive; TN, true negative; TP, true positive; CT, computed tomography; MRI, magnetic resonance imaging; PET/CT, fluorine-18-fluorodeoxyglucose positron emission tomography/computed tomography; CCRT, concurrent chemoradiation therapy; LL, laparoscopic lymphadenectomy; EFRT, extended-field radiation therapy. 
cycles), and supportive care. Data on DMCs were obtained from the hospital database at the Faculty of Medicine Vajira Hospital and the National Cancer Institute of Thailand. DMC data of 59 newly diagnosed LACC patients who were receiving treatment as CCRT or CCRT plus EFRT, 97 patients with no disease and no severe late SEs at follow-up period, and 38 patients with disease recurrence were collected during June to December 2019. However, the cost of management of grades 3-4 of late GI and GU SEs was used from the hospital database because of the small number of these patients. All investigation or treatment costs which were out-of-pocket expenses incurred by patients were included. All charges were converted to costs using a cost-to-charge ratio of $1.63 .^{28}$

DnMCs were the costs incurred by patients and their families due to illness, including the costs of travel, food, informal care, and productivity loss of caregivers. These costs were gathered from interviewing the same patients from whom we collected DMCs using a developed data collection form. Ethical approval was granted by the Institutional
Review Board at both hospitals and Faculty of Pharmacy, Mahidol University. All patients provided informed consent, in accordance with the Declaration of Helsinki. Future costs were adjusted to the present values in 2019 using the discount rate of $3 \%{ }^{29}$ All costs were presented in both Thai Baht (THB) and US\$ using the exchange rate of 32 THB/US\$. All cost parameters are shown in Table 2.

\section{Utility Parameters}

Utility data were collected from interviewing LACC patients during the entire study period along with DnMCs using the EQ-5D-5L (Thai version) questionnaire. ${ }^{30}$ Patients who were selected to be interviewed included 59 patients for newly diagnosed state and receiving treatment, 97 patients for no disease state without severe late SEs, and 38 patients for disease progression state. However, due to a limited number of LACC patients who developed severe GI and GU late SEs during the study period, we assumed that the utility data of these

Table 2 Cost and Utility Parameters

\begin{tabular}{|c|c|c|}
\hline Parameters & Mean (US\$/THB) & Range (US\$/THB) \\
\hline \multicolumn{3}{|l|}{ Direct Medical Costs } \\
\hline CT whole abdomen & $599 / 19,155$ & $449-748 / I 4,367-23,945$ \\
\hline MRI whole abdomen & $917 / 29,340$ & $688-1146 / 22,005-36,675$ \\
\hline $\mathrm{PET} / \mathrm{CT}$ & $3056 / 97,800$ & $2292-3820 / 73,350-122,250$ \\
\hline Laparoscopic lymphadenectomy & $2116 / 67,712$ & I587-2645/50,784-84,640 \\
\hline Concurrent chemoradiation therapy & $4291 / 137,312$ & $3218-5364 / 102,984-171,640$ \\
\hline Extended-field radiation therapy & $4952 / 158,452$ & $37 \mid 4-6190 / 118,839-198,065$ \\
\hline Palliative treatment & $9665 / 309,277$ & $7249-12,08 \mid / 231,958-386,596$ \\
\hline Treatment grades $3-4$ of late $\mathrm{Gl}$ side effect & $6750 / 216,000$ & $5063-8438 / 162,000-270,000$ \\
\hline Treatment grades $3-4$ of late GU side effect & $8558 / 273,86 I$ & $6419-10,698 / 205,396-342,326$ \\
\hline \multicolumn{3}{|l|}{ Direct non-medical costs } \\
\hline \multicolumn{3}{|l|}{ Treatment period } \\
\hline Concurrent chemoradiation therapy & $1033 / 33,063$ & $775-1292 / 24,798-41,330$ \\
\hline Extended-field radiation therapy & $3046 / 97,463$ & $2284-3807 / 73,097-121,828$ \\
\hline \multicolumn{3}{|l|}{ Follow-up period } \\
\hline No disease and no severe side effects & $1113 / 35,629$ & $835-1392 / 26,722-44,536$ \\
\hline No disease with grades $3-4 \mathrm{Gl}$ side effects & $1532 / 49,040$ & || $49-1916 / 36,780-6 \mid, 300$ \\
\hline No disease with grades $3-4 \mathrm{GU}$ side effects & $1253 / 40,096$ & $940-1566 / 30,072-50,121$ \\
\hline Disease progression & $2158 / 69,074$ & $|6| 9-2698 / 5 \mid, 806-86,343$ \\
\hline Utility & & $95 \%$ confidence interval \\
\hline Treatment period to first six months & 0.89 & $0.86-0.92$ \\
\hline \multicolumn{3}{|l|}{ Follow-up period } \\
\hline No disease and no severe side effects & 0.93 & $0.91-0.95$ \\
\hline No disease with grades $3-4 \mathrm{Gl}$ side effects & 0.86 & $0.8 I-0.89$ \\
\hline No disease with grades $3-4 \mathrm{GU}$ side effects & 0.89 & $0.84-0.94$ \\
\hline Disease progression & 0.72 & $0.64-0.80$ \\
\hline
\end{tabular}

Abbreviations: THB, Thai baht; CT, computed tomography; MRI, magnetic resonance imaging; PET/CT, fluorine-I8-fluorodeoxyglucose positron emission tomography/ computed tomography; GI, gastrointestinal system; GU, genitourinary system. 
patients were collected from colorectal cancer and bladder cancer patients, as these patients had the same symptoms as LACC patients with severe GI and GU late SEs. All utility parameters are demonstrated in Table 2 .

\section{Sensitivity Analysis}

One-way sensitivity and probabilistic sensitivity analyses (PSA) were performed using second-order Monte Carlo simulation to examine the uncertainty of the parameters. One-way sensitivity analysis was used to investigate the effect of each parameter value on the change in ICER values. All costs varied in the range of $75 \%-125 \%$, while the ranges of $95 \%$ confidence interval (CI) were used in all probabilities and utility parameters. Tornado diagram was used to present the one-way sensitivity analysis results. PSA was applied to evaluate the change in the ICER values when all parameters were simultaneously varied using the Monte Carlo simulation for 1000 iterations by generating random values for each parameter. The cost-effectiveness acceptability curves were used to present the PSA results. Based on the societal willingness-to-pay (WTP) threshold of Thailand was 160,000 THB or US $\$ 5000,{ }^{28}$ if the ICER of the diagnostic test below the threshold was defined as a costeffective intervention for PALND in LACC patients.

\section{Results}

All results of NoIx and six strategies from three types of diagnostic tests with two approaches in terms of costs from provider and societal perspectives, QALYs, and six ICERs are reported in Table 3. The costs for 5 years of LACC patients with NoIx before treatment were US\$8026 and US\$11,444 from the provider and societal perspectives, respectively, and their QALYs for that corresponding time was 3.70. NoIx was more effective and less costly for LACC patients who had some chance of PALN metastasis or stage IIIC2. When each of the six strategies was compared with NoIx, more additional costs in thee perspective of society were shown with US $\$ 1835$, US\$1735, US\$2022, US\$1987, US\$4002, and US \$4176 per patient for CT without LL, CT with LL, MRI without LL, MRI with LL, PET/CT without LL, and PET/CT with LL, whereas the QALYs were decreased by $0.07,0.08,0.07$, $0.08,0.05$, and 0.07 , respectively. In addition, CT, MRI, and PET/CT yielded the incremental cost of US $\$ 146,832$, US $\$ 159,118$, and US $\$ 148,988$ per PALN case detected compared with no investigation, respectively (Table 4).

When each parameter was explored separately to prove its impact with one-way sensitivity analysis, all parameters still supported the effectiveness of NoIx. The most meaningful parameter which influenced the ICER values to be positive value for using diagnostic tests were DFS for patients who had positive finding at PALN by CT regardless of TP or FP result. When this value was at the lower bar of $95 \% \mathrm{CI}$, the ICER values were increased to about $700 \%$ by decreasing the cost and gaining QALYs for CT with no LL. However, the ICER was still negative (-US\$244,446), because the number of QALYs of CT with no LL from this adjusted value was still less than for NoIx (-0.006). The same situation was expressed for FP results from CT which the changing of DFS was able to provide less additional cost and more QALYs with the small distinction at -0.014 . That created the increasing number of ICER at $270 \%$, but a negative value was shown at -US\$111,441.

Table 3 Outcomes of Cost-Effectiveness Analysis Comparing Seven Approaches (No Investigation and Six Strategies)

\begin{tabular}{|c|c|c|c|c|c|c|c|}
\hline \multirow[t]{2}{*}{ Outcomes } & \multicolumn{7}{|c|}{ Approaches } \\
\hline & $\begin{array}{c}\text { I. No } \\
\text { Investigation }\end{array}$ & $\begin{array}{l}\text { 2. CT without } \\
\mathrm{LL}\end{array}$ & 3. CT with LL & $\begin{array}{l}\text { 4. MRI without } \\
\text { LL }\end{array}$ & $\begin{array}{l}\text { 5. MRI with } \\
\text { LL }\end{array}$ & $\begin{array}{c}\text { 6.PET/CT without } \\
\text { LL }\end{array}$ & $\begin{array}{c}\text { 7.PET/CT with } \\
\text { LL }\end{array}$ \\
\hline \multicolumn{8}{|c|}{ Cost (US\$/THB) } \\
\hline Provider & $8026 / 256,829$ & $9643 / 308,569$ & $9619 / 307,814$ & $9852 / 315,272$ & $9874 / 315,983$ & II,862/379,588 & $|2,05| / 385,624$ \\
\hline Society & $11,444 / 366,202$ & $13,279 / 424,927$ & $13,|79 / 42|, 724$ & $13,466 / 430,918$ & $|3,43| / 429,796$ & $15,446 / 494,270$ & $15,620 / 499,824$ \\
\hline QALY & 3.70 & 3.63 & 3.62 & 3.63 & 3.62 & 3.65 & 3.63 \\
\hline \multicolumn{2}{|c|}{$\begin{array}{l}\text { ICER (US\$ per QALY/THB per } \\
\text { QALY) }\end{array}$} & $\begin{array}{l}-29,679 / \\
-949,740\end{array}$ & $\begin{array}{l}-23,505 / \\
-752,152\end{array}$ & $\begin{array}{c}-32,536 \mid \\
-1,041,160\end{array}$ & $\begin{array}{l}-26,340 / \\
-842,864\end{array}$ & $-87,656 /-2,804,992$ & $\begin{array}{c}-60,586 / \\
-1,938,750\end{array}$ \\
\hline \multicolumn{2}{|l|}{ Dominant } & Dominated & Dominated & Dominated & Dominated & Dominated & Dominated \\
\hline
\end{tabular}

Abbreviations: CT, computed tomography; MRI, magnetic resonance imaging; PET/CT, fluorine-18-fluorodeoxyglucose positron emission tomography/computed tomography; LL, laparoscopic lymphadenectomy; THB, Thai baht; QALY, quality-adjusted life year; ICER, incremental cost-effectiveness ratio. 
Table 4 Outcomes of Cost-Effectiveness Analysis in Terms of Cost per One Case of Para-Aortic Lymph Node Detection

\begin{tabular}{|l|c|c|c|}
\hline Outcomes (US $\$$ /THB) & CT & MRI & PET/CT \\
\hline Incidence of PALN metastasis of 16\% & & $167, \mid 44 / 5,348,619$ & $157,014 / 5,024,438$ \\
- Cost per case detected & $154,858 / 4,955,443$ & $159,118 / 5,091,789$ & $148,988 / 4,767,609$ \\
- ICER & $146,832 / 4,698,614$ & & $83,74 \mid / 2,679,700$ \\
\hline Incidence of PALN metastasis of 30\% & & $89,114 / 2,852,597$ & $75,7|5 / 2,422,87|$ \\
- Cost per case detected & $82,591 / 2,642,903$ & $81, \mid 18 / 2,595,767$ & \\
- ICER & $74,565 / 2,386,074$ & & \\
\hline
\end{tabular}

Abbreviations: PALN, para-aortic lymph node; CT, computed tomography; MRI, magnetic resonance imaging; PET/CT, fluorine-18-fluorodeoxyglucose positron emission tomography/computed tomography.

Therefore, NoIx was a dominant approach because of more cost saving and more QALYs. For other parameters including discount rate, incidence rate of PALN metastasis, performance of each diagnostic test, DMCs, DnMCs, other treatment outcomes, and utilities, if these were changed in the range as described above, there was no significant effect on ICER. The first ten parameters which had strong effect on ICER are presented in a Tornado diagram in Figure 4. Although the incidence of PALN metastasis was increased to $30 \%$, the ICER values in terms of cost per PALN case detected were much higher compared to NoIx (Table 4).

Figure 5 demonstrates the probabilities of costeffectiveness between NoIx and six strategies of three diagnostic tests when WTP threshold was concerned. NoIx was presented to be the most cost-effective at the probability of $82.0 \%$. The probabilities of cost- effectiveness for CT without LL, CT with LL, MRI without LL, and MRI with LL were at $9.4 \%, 8.2 \%, 0.3 \%$, and $0.1 \%$, respectively, whereas PET/CT without LL and PET/ CT with LL were not shown to be of value for PALND in LACC patients in the Thai context. Figure 6 shows the cost-effectiveness plane comparing NoIx and all other six strategies; almost all ICER values of NoIx were located in the right lower quadrant, whereas most ICER values of other strategies were placed in the left upper quadrant and above the WTP line.

\section{Discussion}

During the last five decades, over $90 \%$ of studies about economic analysis in cervical cancer were concentrated on screening or vaccination programs. A few studies were interested in cervical cancer treatment, ${ }^{31-36}$ while only

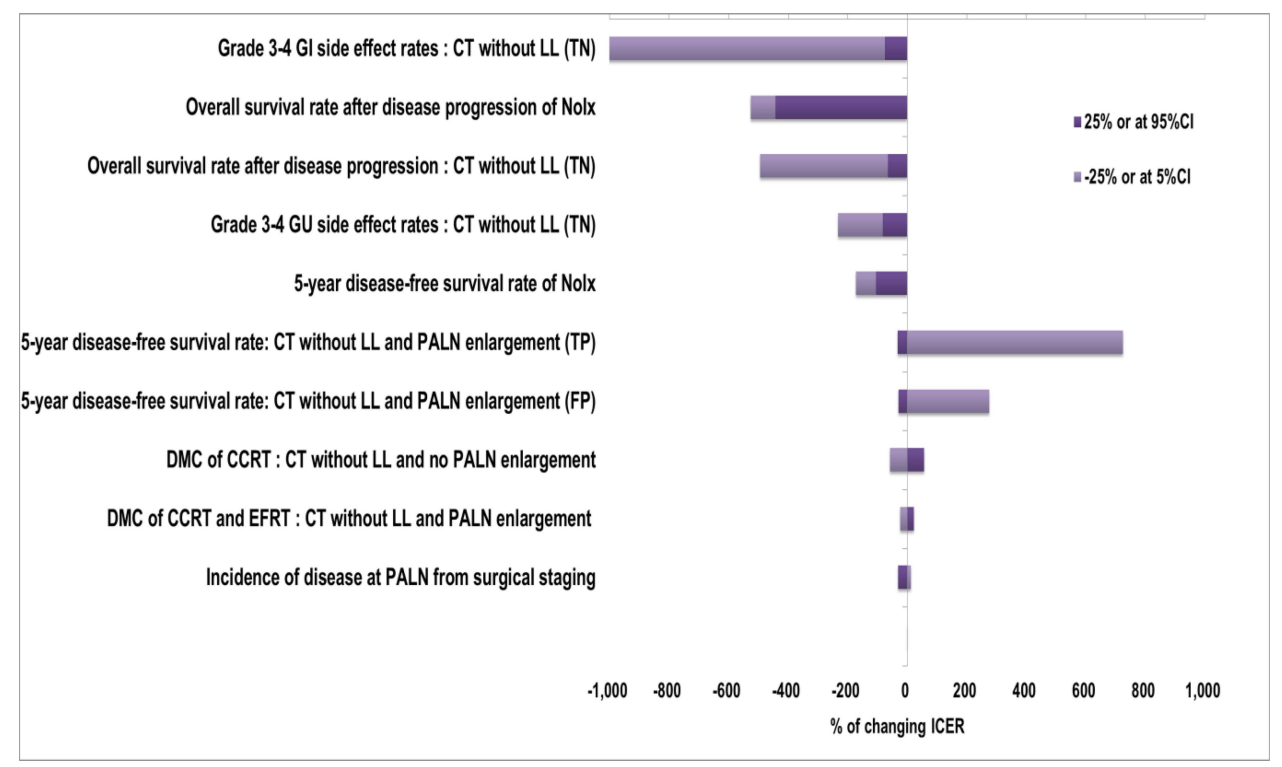

Figure 4 One-way sensitivity analysis (Tornado diagram) for the top ten most influential parameters.

Abbreviations: GI, gastrointestinal; GU, genitourinary; CT, computed tomography; LL, laparoscopic lymphadenectomy; PALN, para-aortic lymph node; FP, false positive; TN, true negative; TP, true positive; DMC, direct medical cost; CCRT, concurrent chemoradiation; EFRT, extended-field radiation therapy; Nolx, no investigation; ICER, incremental cost-effectiveness ratio; $\mathrm{Cl}$, confidence interval. 


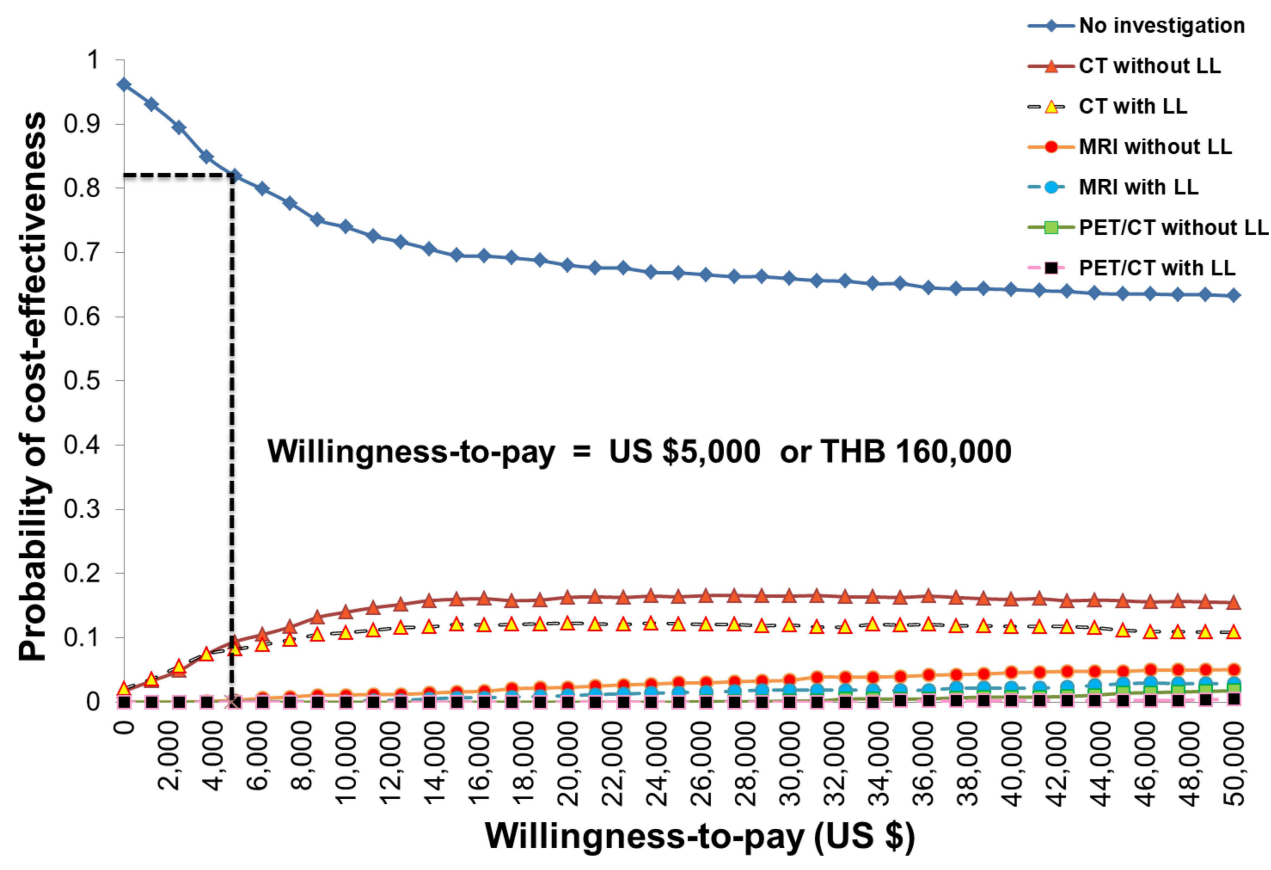

Figure 5 Cost-effectiveness acceptability curves of using different approaches for para-aortic lymph nodes detection in locally advanced cervical cancer patients with a willingness-to-pay threshold of Thailand.

Abbreviations: CT, computed tomography; MRI, magnetic resonance imaging; PET/CT, fluorine-I8-fluorodeoxyglucose positron emission tomography/computed tomography; LL, laparoscopic lymphadenectomy.

one study explored the additional value of para-aortic lymphadenectomy before starting treatment. ${ }^{31}$ Currently, advanced cervical cancer investigations are needed to diagnose and determine severity of disease before starting treatment; however, these technologies are commonly costly. Given that the majority of cervical cancer patients were in underdeveloped and developing countries that lack healthcare resources and usually comply with all guidelines or recommendations from well-resourced countries, cost-effectiveness information of cervical cancer investigations in their context would be very helpful information for policy decision making and clinical practice. To date, this study is the first to evaluate the cost-effectiveness of diagnostic tests for PALN detection in LACC patients compared with NoIx. The results from this study would provide the information on common practice of LACC patients at the diagnostic time, whether using special diagnostic tests could increase value for money.

The major disadvantage of clinical staging is that it is unable to detect disease at the para-aortic lymph node. The number of patients who would have missed the proper treatment at para-aortic lymph node was about $15.6 \%$ (98/621 patients), according to the study by Berman et al. ${ }^{4}$ In our study, we presented the final outcomes of clinical stage which included the results of undertreatment at para-aortic lymph node in patients with stage IIIC2. Nevertheless, the number of overtreated patients due to inaccuracy from clinical staging might not be available, because all LACC patients would receive radiation therapy at the whole pelvis and concurrence with chemotherapy as a standard treatment. Our results suggested that NoIx or receiving basic clinical staging was more effective and less costly before initiating treatment for LACC patients compared to CT, MRI, and PET/CT. The additional value of using special diagnostic tests was not evident in terms of cost-effectiveness. The obvious reason of this was that all the diagnostic tests did not have the capability to gain more QALYs compared to NoIx. Although the additional cost of each approach was not a large amount of money, enormous negative effects on ICERs appeared. In the context of Thailand with the threshold of WTP of US $\$ 5000$, real clinical staging by pelvic examination was a dominant option in LACC patients.

The result of this study may not be directly compared with previous study because no study has ever explored this topic before. Nevertheless, the results from Lee et al's study ${ }^{31}$ were quite similar to our study. They reported the cost-effectiveness of proven disease at PALN by surgery in case negative finding of PET/CT at PALN was identified. Incremental cost per QALY gained from using LL 


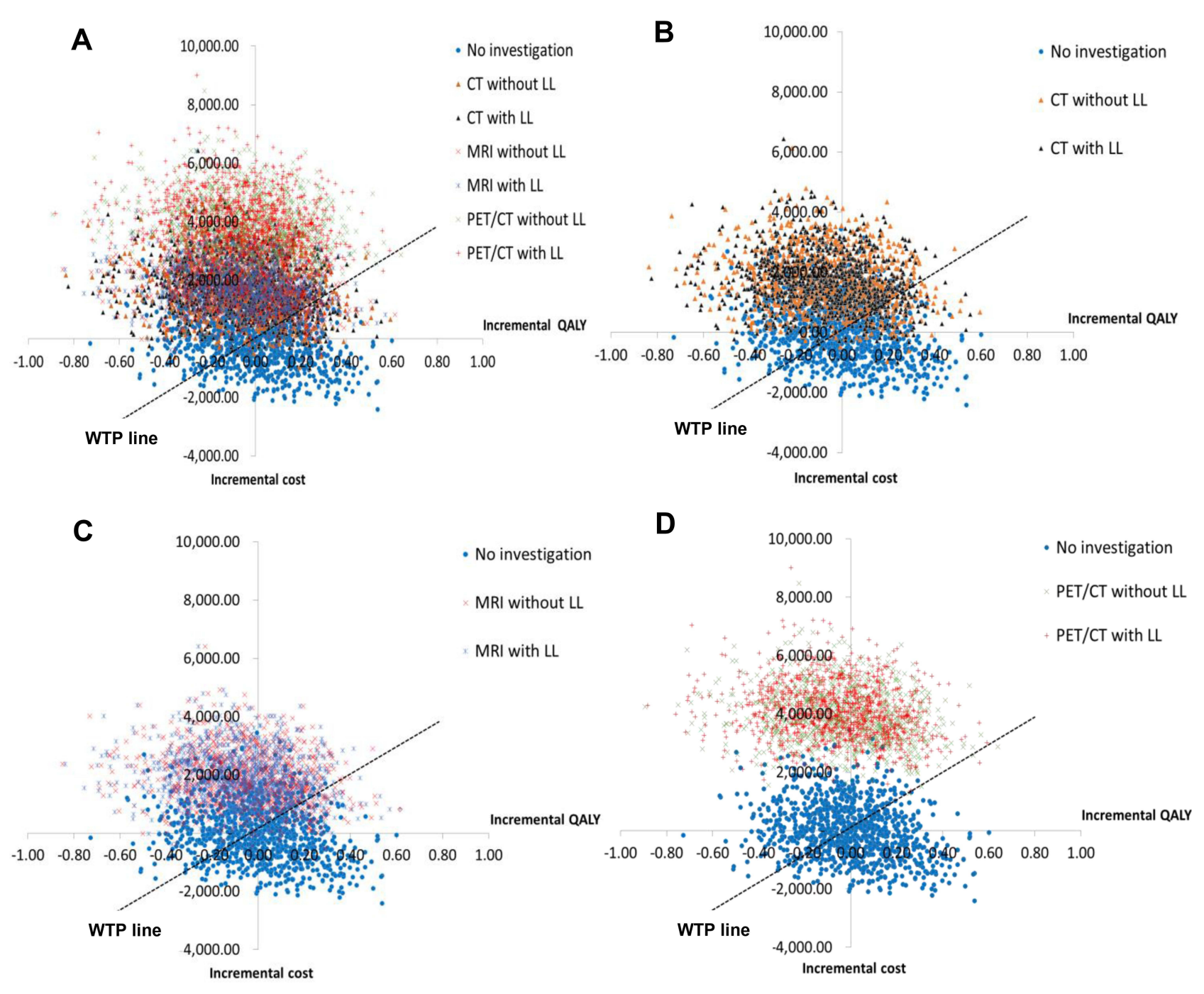

Figure 6 Cost-effectiveness planes with the threshold willingness-to-pay (WTP) of US\$5000 or THB 160,000 per quality-adjusted life year for all approaches in (A), comparing no investigation and CT in (B), comparing no investigation and MRI in (C), and comparing no investigation and PET/CT in (D).

Abbreviations: CT, computed tomography; MRI, magnetic resonance imaging; PET/CT, fluorine-l8-fluorodeoxyglucose positron emission tomography/computed tomography; LL, laparoscopic lymphadenectomy; QALY, quality-adjusted life year; WTP, willingness-to-pay.

compared with no surgery was US\$19,505 per QALY. However, Lee et $\mathrm{al}^{31}$ obtained almost all utility parameters from another study which related to prostate $\operatorname{cancer}^{37}$ or post-operative CCRT for early stage cervical cancer. ${ }^{38}$ Additionally, more variation of tools for analyzing utility was observed. ${ }^{37-40}$ Using the important parameter from heterogeneous sources might make for the different outcomes, and thus results could not be compared with this study. In contrast, the utility data in the current study were collected from interviewing LACC patients in all health states by the standard tool EQ-5D-5L, and this was the strength of our study.

According to cost-effectiveness acceptability curves, CT without LL was the next approach that had the probability of effectiveness at $9.4 \%$ for ceiling ratio of
Thailand. One-way sensitivity analysis results suggested that the most influential parameter for ICER to be a positive value was the survival outcome of patients who had suspected disease at PALN by CT. If the number of these outcomes had increased to the value of $95 \% \mathrm{CI}$, the ICER was changed to approximately $700 \%$ for TP. These results were robust, supporting that the effective treatment strategies for patients with disease at PALN was more significant than knowing the status of this node to correct stage following the new FIGO staging system. In addition, the incidences of PALN metastasis $(16 \%),{ }^{4}$ with the range of $95 \%$ CI of $13 \%$ to $18 \%$, did not have much impact on changing ICERs (-9\% to $-12 \%)$. In other words, if incidence of PALN was increased, the value of $\mathrm{CT}$ or other diagnostic tests was decreased. The 
reason might be because the high number of $\mathrm{FN}$ was an important part of treatment failure. Based on the largest meta-analysis study of Liu, the mean of FN for CT, MRI, and PET/CT was $43 \%, 46 \%$, and $34 \%$, respectively. ${ }^{24}$ This implied that about one-third to half of all patients did not have a benefit from these diagnostic tests, leading to lower effectiveness.

When all parameters were varied together, NoIx generated the probability to be the most effective approach even though WTP threshold would be increased to 10 times the ceiling threshold in Thailand. One crucial reason for the powerlessness of using diagnostic tests was that they were unable to improve the QALYs from the consequence of PALND. If QALY was not considered, and cost per one case of PALN detected from all diagnostic tests was calculated. The high costs and high ICERs of all investigations were found because the moderate sensitivity for detection of PALN was still the obvious problem.

There were some limitations in this study that need to be addressed. Firstly, since 1999, CCRT has been accepted to be standard treatment for LACC patients, and some diagnostic tests before treatment were offered for them simultaneously. Consequently, the parameter for NoIx apart from clinical staging in the period of modern treatment was hardly found. One of our retrospective studies about using carboplatin weekly was used for this condition. ${ }^{25}$ The outcome in terms of 5-year DFS $(63 \%)^{25}$ was lower than the study using diagnostic test to exclude patients with PALN enlargement (65\%). ${ }^{26}$ Thus, it was quite reasonable for applying in the model. Secondly, as a major disadvantage of clinical staging by pelvic examination or NoIx was that it is unable to detect disease at the para-aortic lymph node, and there has been no study investigating the sensitivity and specificity of clinical staging, we did not consider these parameters in our model. This could lead to the underestimation of the effectiveness of NoIx. Even though we did not consider the effectiveness of NoIx, our results suggested that NoIx was a dominant option compared to CT, MRI, and PET/ CT. Thirdly, as there has been no study investigating the sensitivity and specificity of LL for proof of the disease at enlarged para-aortic lymph node compared with laparotomy or open surgery approach, we did not include this parameter in our model. Future study should further investigate this parameter. Additionally, the probability of SEs from EFRT could not be obtained from original study. ${ }^{17}$ Due to the distinct period of time, variation of radiation therapy technique was apparent. The number of late GI and GU SEs reported from the study by Jung et $\mathrm{al}^{17}$ was very small and lower than that of CCRT at whole pelvis. This was unreasonable, thus we assumed the same SEs as CCRT. The technique of CCRT used in this study was two-dimensional radiation therapy which was the conventional technique from our previous study $^{25}$ and the study by Pearcey et al. ${ }^{26}$ Therefore, the long-term GI and GU SEs were rather high. Modern radiation therapy techniques such as 3D-CRT or intensity-modulated radiation therapy might produce a lower number of these SEs, but more cost was unavoidable. These modern techniques should be used for effectiveness analysis in future studies. Moreover, the sample size of patients in each health state was limited, especially patients who received LL and EFRT. More cervical cancer patients in stage IIIC2 patients, both IIIC2 $r$ and IIIC $2 p$, need to be included for more valid and reliable results for this issue.

\section{Conclusion}

NoIx or receiving basic clinical staging was a dominant option (more effective and less costly) before initiating treatment for LACC patients when compared with three common diagnostic tests including CT, MRI, and PET/CT for PALND. However, these investigations are able to add more value if more effective treatments for disease at PALN or stage IIIC2 are available in the future prospective trials.

\section{Data Sharing Statement}

The datasets generated during and/or analyzed during the current study are available upon reasonable request.

\section{Acknowledgments}

This study was a part of $\mathrm{PhD}$ thesis of Kanyarat Katanyoo at Social, Economic and Administrative Pharmacy (SEAP) Graduate Program, Faculty of Pharmacy, Mahidol University. We would like to express our thanks to the EuroQoL group for allowing us to use the EQ-5D questionnaires. We thank all patients and their families who participated in the data collection process.

\section{Author Contributions}

All authors made substantial contributions to conception and design, acquisition of data, analysis and interpretation of data, took part in drafting the article and revising it critically for important intellectual content. All authors agreed to submit to the current journal, gave final approval of the version for publication and any significant changes 
introduced at the proofing stage; and agree to take responsibility and be accountable for the contents of the article.

\section{Disclosure}

The authors report no conflicts of interest in this work.

\section{References}

1. International Agency for Research on Cancer; 2018. Available from: http://globocan.iarc.fr. Accessed October 8, 2018.

2. Gakidou E, Nordhagen S, Obermeyer Z. Coverage of cervical cancer screening in 57 countries: low average levels and large inequalities. PLoS Med. 2008;5:e132. doi:10.1371/journal.pmed.0050132

3. Bhatla N, Aoki D, Sharma DN, Sankaranarayanan R. FIGO cancer report 2018. Cancer of the cervix uteri. Int J Gynecol Obstet. 2018;143(Suppl. 2):22-36. doi:10.1002/ijgo.12611

4. Berman ML, Keys H, Creasman W, Disaia P, Bundy B, Blessing J. Survival and patterns of recurrence in cervical cancer metastatic to periaortic lymph nodes (a Gynecologic Oncology Group study). Gynecol Oncol. 1984;19:8-16. doi:10.1016/0090-8258(84)90151-3

5. Piver MS, Barlow JJ. Para-aortic lymphadenectomy in staging patients with advanced local cervical cancer. Obstet Gynecol. 1974;43:544-548.

6. Whitney CW, Sause W, Bundy BN, et al. Randomized comparison of fluorouracil plus cisplatin versus hydroxyurea as an adjunct to radiation therapy in stage IIB-IVA carcinoma of the cervix with negative para-aortic lymph nodes: a Gynecologic Oncology Group study. J Clin Oncol. 1999;17:1339-1348. doi:10.1200/JCO.1999.17.5.1339

7. Rose PG, Bundy BN, Watkins EB, et al. Concurrent cisplatin-based radiotherapy and chemotherapy for locally advanced cervical cancer. $N$ Engl J Med. 1999;340:1144-1153. doi:10.1056/NEJM199904 153401502

8. Lanciano R, Calkins A, Bundy BN, et al. Randomized comparison of weekly cisplatin or protracted venous infusion of fluorouracil in combination with pelvic radiation in advanced cervix cancer: a gynecologic oncology group study. $J$ Clin Oncol. 2005;23:8289-8295. doi:10.1200/JCO.2004.00.0497

9. Capozzi VA, Sozzi G, Monfardini L, et al. Transperitoneal versus extraperitoneal laparoscopic aortic lymph nodal staging for locally advanced cervical cancer: a systematic review and meta-analysis. Eur J Surg Oncol. 2021;47:2256-2264.

10. Negra o MV, Silva Lima JM, Severino Silva SC, Estevez Diz MDP, Costa Miranda V. Management of para-aortic lymph node disease in patients with cervical cancer. What is the best approach? Int J Gynecol Cancer. 2017;27:543-549. doi:10.1097/IGC.0000000000000914

11. Hwang L, Bailey A, Lea J, Albuquerque K. Para-aortic nodal metastases in cervical cancer: a blind spot in the International Federation of Gynecology and Obstetrics staging system: current diagnosis and management. Future Oncol. 2015;11:309-322. doi:10.2217/fon.14.200

12. Lai $\mathrm{CH}$, Huang $\mathrm{KG}$, Hong $\mathrm{JH}$, et al. Randomized trial of surgical staging (extraperitoneal or laparoscopic) versus clinical staging in locally advanced cervical cancer. Gynecol Oncol. 2003;89:160-167. doi:10.1016/S0090-8258(03)00064-7

13. Jang H, Chun M, Cho O, Heo JS, Ryu HS, Chang SJ. Prognostic factors and treatment outcome after radiotherapy in cervical cancer patients with isolated para-aortic lymph node metastases. $J$ Gynecol Oncol. 2013;24:229-235. doi:10.3802/jgo.2013.24.3.229

14. Stryker JA, Mortel R. Survival following extended field irradiation in carcinoma of cervix metastatic to para-aortic lymph nodes. Gynecol Oncol. 2000;79:399-405. doi:10.1006/gyno.2000.5977

15. Shim SH, Kim DYK, Lee SJ, et al. Prediction model for para-aortic lymph node metastasis in patients with locally advanced cervical cancer. Gynecol Oncol. 2017;144:40-45. doi:10.1016/j. ygyno.2016.11.011
16. Kim YS, Kim JH, Ahn SD, et al. High-dose extended-field irradiation and high-dose-rate brachytherapy with concurrent chemotherapy for cervical cancer with positive para-aortic lymph nodes. Int $J$ Radiat Oncol Biol Phys. 2009;74:1522-1528. doi:10.1016/j.ijrobp.2008.10.024

17. Jung J, Park G, Kim YS. Definitive extended-field intensity-modulated radiotherapy with chemotherapy for cervical cancer with para-aortic nodal metastasis. Anticancer Res. 2014;34:4361-4366.

18. Yoon HI, Cha J, Keum KC, et al. Treatment outcomes of extended-field radiation therapy and the effect of concurrent chemotherapy on uterine cervical cancer with para-aortic lymph node metastasis. Radiat Oncol. 2015;10:18. doi:10.1186/s13014-014-0320-5

19. Marnitz S, Schram J, Budach V, et al. Extended field chemoradiation for cervical cancer patients with histologically proven para-aortic lymph node metastases after laparoscopic lymphadenectomy. Strahlenther Onkol. 2015;191:421-428. doi:10.1007/s00066-014-0785-z

20. Small W, Winter K, Levenback C, et al. Extended-field irradiation and intracavitary brachytherapy combined with cisplatin chemotherapy for cervical cancer with positive para-aortic or high common iliac lymph nodes: results of ARM 1 of RTOG 0116. Int J Radiat Oncol Biol Phys. 2007;68:1081-1087. doi:10.1016/j.ijrobp.2007.01.026

21. Rotman M, Pajak TF, Choi K, et al. Prophylactic extended-field irradiation of para-aortic lymph nodes in stages IIB and bulky IB and IIA cervical carcinomas. Ten-year treatment results of RTOG 79-20. JAMA. 1995;274:387-393. doi:10.1001/jama.1995.03530050035029

22. Whitley NO, Brenner DE, Francis A, et al. Computed tomographic evaluation of carcinoma of the cervix. Radiology. 1982;142:439-446. doi:10.1148/radiology.142.2.7054834

23. Kitagawa R, Katsumata K, Shibata T, et al. Paclitaxel plus carboplatin versus paclitaxel plus cisplatin in metastatic or recurrent cervical cancer: the open label randomized phase III trial (JCOG0505). J Clin Oncol. 2015;33:2129-2135. doi:10.1200/JCO.2014.58.4391

24. Liu B, Gao S, Li S. A comprehensive comparison of CT, MRI, Positron Emission Tomography or Positron Emission Tomography/ $\mathrm{CT}$, and diffusion weighted imaging-MRI for detecting the lymph nodes metastases in patients with cervical cancer: a meta-analysis based on 67 studies. Gynecol Obstet Invest. 2017;82:209-222. doi:10.1159/000456006

25. Katanyoo K, Tangitgamol S, Chongthanakorn M, et al. Treatment outcomes of concurrent weekly carboplatin with radiation therapy in locally advanced cervical cancer patients. Gynecol Oncol. 2011;123:571-576. doi:10.1016/j.ygyno.2011.09.001

26. Pearcey R, Brundage M, Drouin P, et al. Phase III trial comparing radical radiotherapy with and without cisplatin chemotherapy in patients with advanced squamous cell cancer of the cervix. $J$ Clin Oncol. 2002;20:966-972. doi:10.1200/JCO.2002.20.4.966

27. Life table by country-Thailand; 2020. Available from: https://apps. who.int/gho/data/view.main.61640?lang=en. Accessed February 29, 2020.

28. Riewpaiboon A. Cost analysis. In: Chaikledkaew U, Teerawattananon Y, editors. Thai National Health Technology Assessment Guidelines. 2nd ed. Nonthaburi: Watcharin PP; 2013:23-42.

29. Permsuwan U, Guntawongwan K, Buddhawongsa P. Handling time in economic evaluation studies. J Med Assoc Thai. 2014;97(Suppl 5):S50-8.

30. Pattanaphesaj J, Thavorncharoensap M, Ramos-Goñi JM, Tongsiri S, Ingsrisawang L, Teerawattananon Y. The EQ-5D-5L Valuation study in Thailand. Expert Rev Pharmacoecon Outcomes Res. 2018;18:551-558. doi:10.1080/14737167.2018.1494574

31. Lee JY, Kim Y, Lee TJ, et al. Cost-effectiveness of para-aortic lymphadenectomy before chemoradiotherapy in locally advanced cervical cancer. J Gynecol Oncol. 2015;26:171-178. doi:10.3802/ jgo.2015.26.3.171

32. Rajan N, Barraclough H, Puri T, Orlando M. Cost effectiveness of concurrent gemcitabine and cisplatin and radiation followed by adjuvant gemcitabine and cisplatin in stages IIB-IVA cervical cancer. Gynecol Oncol Rep. 2014;10:36-37. doi:10.1016/j.gore.2014.09.002 
33. Kim H, Rajagopalan MS, Beriwal S, Huq MS, Smith KJ. Costeffectiveness analysis of 3D image-guided brachytherapy compared with 2D brachytherapy in the treatment of locally advanced cervical cancer. Brachytherapy. 2015;14:29-36. doi:10.1016/j.brachy.2014.09.002

34. Chai Y, Wang J, Wang T, et al. Cost-effectiveness of radical hysterectomy with adjuvant radiotherapy versus radical radiotherapy for FIGO stage IIB cervical cancer. Onco Targets Ther. 2016;9:349-354. doi:10.2147/OTT.S90798

35. Perdrizet J, D'Souza D, Skliarenko J, et al. A cost-utility analysis of magnetic resonance (MR) guided brachytherapy versus two-dimensional and computed tomography (CT) guided brachytherapy for locally advanced cervical cancer. Int J Radiat Oncol Biol Phys. 2020;107:512-521. doi:10.1016/j.ijrobp.2020.03.004

36. Lesnock JL, Farris C, Beriwal S, Krivak TC. Upfront treatment of locally advanced cervical cancer with intensity modulated radiation therapy compared to four-field radiation therapy: a cost-effectiveness analysis. Gynecol Oncol. 2013;129:574-579. doi:10.1016/j. ygyno.2013.02.012
37. Konski A, Watkins-Bruner D, Feigenberg S, et al. Using decision analysis to determine the cost-effectiveness of intensity-modulated radiation therapy in the treatment of intermediate risk prostate cancer. Int J Radiat Oncol Biol Phys. 2006;66:408-415. doi:10.1016/j. ijrobp.2006.04.049

38. Jewell EL, Smrtka M, Broadwater MS, et al. Utility scores and treatment preferences for clinical early stage cervical cancer. Value Health. 2011;14:582-586. doi:10.1016/j.jval.2010.11.017

39. El-Gazzaz G, Hull TL, Mignanelli E, Hammel J, Gurland B, Zutchi M. Obstetric and cryptoglandular rectovaginal fistulas: long-term surgical outcome, quality of life, and sexual function. J Gastrointest Surg. 2010;14:1758-1763. doi:10.1007/s11605-010-1259-y

40. Ernst EJ, Ernst ME, Hoehns J, Bergus GR. Women's QOL is decreased by acute cystitis and antibiotic adverse effects associated with treatment. Health Qual Life Outcomes. 2005;3:45. doi:10.1186/1477-7525-3-45
ClinicoEconomics and Outcomes Research

\section{Publish your work in this journal}

ClinicoEconomics and Outcomes Research is an international, peerreviewed open-access journal focusing on Health Technology Assessment, Pharmacoeconomics and Outcomes Research in the areas of diagnosis, medical devices, and clinical, surgical and pharmacologica intervention. The economic impact of health policy and health systems
Dovepress

organization also constitute important areas of coverage. The manuscript management system is completely online and includes a very quick and fair peer-review system, which is all easy to use. Visit http://www.dovepress.com/testimonials.php to read real quotes from published authors. 